Fecha de recepción: 09-10-2017

Fecha de aceptación: 19-01-2018

Link para este artículo: http://dx.doi.org/10.14198/ALEUA.2018.29-30.11

Puede citar este artículo como:

Payá Beltrán, José, «Alfonso Paso y el teatro universitario», Anales de Literatura Española, n. ${ }^{\circ}$ 29-30

(2018), pp. 231-243

\title{
ALFONSO PASO Y EL TEATRO UNIVERSITARIO
}

\author{
JOSÉ PAYÁ BELTRÁN
}

\section{Resumen}

El presente artículo da cuenta de las nueve comedias de Alfonso Paso, dos de ellas en colaboración, estrenadas entre 1945 y 1956 por el Teatro Español Universitario, así como por diversos grupos teatrales afines al ambiente universitario (Arte Nuevo, TUDE, TPU). En estas piezas advertimos ya algunas de las características que definirán su posterior producción: trazos de machismo, rechazo del Existencialismo francés y tendencia a la crítica social bajo la pátina del humor.

Palabras clave: Alfonso Paso. Teatro. Teatro Universitario. Arte Nuevo. Postguerra.

\begin{abstract}
This article deals with the Alfonso Paso's nine plays - two of them written in colaboration - that were performed for the first time between 1945 and 1956 by the Spanish University Theatre (TEU), and by other theatrical groups related to the University atmosphere (Arte Nuevo, TUDE, TPU). In these plays we can already see some features that will define his later production: lines of male chauvinism, rejection of French Existencialism, and an inclination towards social criticism under the patina of humour.
\end{abstract}

Keywords: Alfonso Paso. Theatre. University Theatre. Arte Nuevo. Post-war

Si tuviéramos que emplear un único adjetivo para calificar la relación que Alfonso Paso mantuvo con el teatro universitario, este adjetivo sería fluctuante. Entre 1945, fecha de la fundación del grupo Arte Nuevo, y 1956, fecha de Una bomba llamada Abelardo, Alfonso Paso estrenó dentro del ambiente universitario madrileño nueve comedias, dos de ellas escritas en colaboración, y todas ellas, excepto la última, en un acto.

Al concluir el Bachillerato, Alfonso Paso intentó, alentado por su madre, la actriz Juana Gil, comenzar la carrera de ingeniero aeronáutico. Tal y como

Anales, 29-30 (2018), pp. 231-243

DOI: 10.14198/ALEUA.2018.29-30.11 
el mismo autor cuenta al crítico Alfredo Marqueríe, a la sexta clase su amigo y compañero desde la infancia, Alfonso Sastre, lo sacó del aula y lo condujo a una taberna cercana donde se tomó un coñac y decidió que no iba a entrar nunca más. Tras este primer fracaso universitario, Alfonso Paso de nuevo probó suerte en otras ramas del saber, pero la terrible asignatura de Matemáticas le cerró cualquier posibilidad de continuar por la vía de las denominadas Ciencias.

Finalmente, fue su padre, don Antonio Paso, quien se impusiera sobre la teoría materna de la «solución práctica», permitiendo que su hijo iniciase la carrera de Filosofía y Letras en la Universidad Complutense de Madrid, donde se licenció en 1951, con premio extraordinario, dentro de la especialidad de Historia de América.

Durante los años universitarios, Alfonso Paso, como otros jovencísimos autores de la época, se aproximó a los grupos teatrales que, desde 1940, habían comenzado a aparecer vinculados a las universidades españolas y al SEU.

Aunque no suele cobijarse bajo las siglas del TEU, la aparición del grupo Arte Nuevo en 1945 se erige como un hito interesantísimo dentro del teatro español de la postguerra, pues supuso el primer intento sólido de hacer un teatro radicalmente diferente del que predominaba en los escenarios españoles.

Compañeros en los estudios de Bachillerato en la Academia Menéndez y Pelayo, Alfonso Paso, el futuro actor Enrique Cerro, Carlos José Costas, Alfonso Sastre y Medardo Fraile fueron los creadores de una serie de piezas teatrales que pretendían llevar a los escenarios. Con este propósito, en el verano de 1945, entran en contacto con el sobrino de Alfonso Paso, José Gordón, mayor que todos ellos y gran aficionado al arte escénico, que los anima a formar una agrupación cultural.

La reunión fundacional del grupo tiene lugar en el Café Arizona, ubicado en la madrileña calle Aguilera, el día 16 de septiembre de ese mismo año.

Poco después de la fundación del grupo se unen los incipientes actores y actrices Encarna Paso y Miguel Narros; y los también creadores José María Palacio, José María de Quinto y José Franco. Este último, por cierto, había trabajado ya durante la II República en el Teatro Escuela de Arte (TEA) y sería el encargado de dirigir la mayoría de las obras.

Había tenido lugar la génesis de uno de los primeros experimentos teatrales que intentarían insuflar un aliento de frescura al decepcionante escenario español: el grupo de teatro independiente Arte Nuevo, cuya «sede»—según lo cuenta Medardo Fraile (Fraile, 1994: 18) — se instaló en «los altos de un gimnasio propiedad de un hermano de Paso, que cedió el local para reuniones y ensayos con generosidad nada común». La cuota de los escasos socios de la agrupación ascendió a diez pesetas al mes. 
A este respecto, en 1964, Alfonso Sastre escribió:

Arte Nuevo surgió como una forma — quizá tumultuosa y confusa— de decir «no» a lo que nos rodeaba; y lo que nos rodeaba, a nosotros que sentíamos la vocación del teatro, era precisamente el teatro que se producía en nuestros escenarios. Si algo nos unía a nosotros, que éramos tan diferentes, era precisamente eso: la náusea ante el teatro burgués de aquel momento: el Benavente póstumo (en contradicción con la persona viva de Benavente), el melodrama galaico-plorante de Torrado, las barbaridades del pos-astracán y los espectáculos pseudofolklóricos, por no contar las débiles supervivencias del teatro versificado (Marquina) y otras dolorosas pruebas del vacío (apud Ruiz Ramón, 1971: 418). ${ }^{1}$

Asimismo, José María de Quinto (en García Lorenzo 1999: 68) recordaba que ninguno de los componentes del grupo se planteaba problemas políticos. «Los únicos problemas que nos preocupaban por aquel entonces eran de índole ética y estética»

El grupo Arte Nuevo llevó a cabo ocho sesiones teatrales, más otras cuatro que podríamos denominar sesiones truncadas. En total, se representaron veintitrés obras.

La vinculación universitaria del nuevo grupo fue evidente, pues más de dos terceras partes de sus componentes estudiaban entonces en la universidad madrileña, y cabe suponer que no entraron en el circuito universitario como una muestra más de su afán rupturista; pues los TEU, pese a gozar de una libertad que las compañías profesionales no tenían, también estaban plegados a los dictámenes del SEU y a las consignas del Régimen, que, en algunos momentos, los utilizó con fines propagandísticos.

En 1949, cuando ya la agrupación había dejado de existir, José Gordón puso un digno colofón a la empresa con la publicación del volumen Teatro de vanguardia. 15 obras de Arte Nuevo, con un prólogo de Alfredo Marqueríe - uno de los mayores defensores del proyecto- y un estudio de Antonio Rodríguez de León (crítico teatral en el diario España bajo el pseudónimo de Sergio Nervo). Gracias a este empeño editorial, hoy podemos leer y analizar unas piezas teatrales que, de otro modo, se hubieran perdido.

La primera obra que se representó, en el teatro Infanta Beatriz, y en sesión única, fue Un tic-tac de reloj, de José Gordón y Alfonso Paso. En esa primera sesión también subieron al escenario: Armando y Julieta, de José María Palacio; y Ha sonado la muerte, de Alfonso Sastre y Medardo Fraile.

1. Las palabras de Alfonso Sastre están tomadas de Alfonso Sastre, Teatro, ed. Taurus, 1964. pp. 55-6, según indica Francisco Ruiz Ramón.

Anales, 29-30 (2018), pp. 231-243 
La comedia en un acto Un tic-tac de reloj se estrenó el 31 de enero de 1946. Los ya mencionados Miguel Narros y Enrique Cerro fueron dos de sus protagonistas. La obra defendía la tesis sobre la capacidad creativa de la juventud frente al anquilosamiento de la madurez y la vejez.

Tras el estreno, las críticas de Sánchez Camargo (El Alcázar), Alfredo Marqueríe $(A B C)$ y Ariel (Marca) fueron, en términos generales, favorables, haciendo hincapié en la valentía de los componentes del grupo y en lo interesante que podían ser estos proyectos cuyos integrantes, en palabras de Marqueríe, «quieren entretener y hacer pensar al mismo tiempo» (De Paco, 1989: 1.069).

Observamos algunos temas que serán recurrentes en las posteriores comedias de Alfonso Paso de su primera etapa: la defensa de la juventud; la crítica a una férrea educación paterna que impide el desarrollo libre del joven; la denuncia de la vida hogareña como gris y apática; y, por último, la convicción de que dicha mediocridad doméstica es el germen de los celos y de las desavenencias entre el matrimonio.

Desde una perspectiva actual, Un tic-tac de reloj es una obra ingenua, que destila buenas intenciones. Para John London (1997: 180) la pieza anticipa algunos de los temas de la dramaturgia de Buero Vallejo, como la participación del espectador al ser aludido por el personaje y también el elemento metateatral en que se enmarca la pieza de modo que resulta más fácil aceptar ciertos diálogos ridículamente sentimentales. La influencia, desde luego, provenía de Nuestra ciudad, de Thornton Wilder, estrenada en el teatro María Guerrero en 1944.

El día 11 de abril de 1946, se estrenó, de nuevo en el teatro Infanta Beatriz, la comedia en un acto, Un día más, escrita en colaboración con Medardo Fraile. El peso de la obra recae esta vez en un personaje femenino, el interpretado por Amparito Conde. Los actores Enrique Cerro, Aníbal Vela y Justo Sanz aparecen de nuevo. Los autores defienden la tesis de que el amor y la ilusión vencen a la idea práctica y materialista de la vida. Para ello sitúan la acción en Nueva York y construyen un mosaico donde un par de parejas y algunos personajes individuales desfilan por el escenario en defensa de la tesis propuesta.

La idea de los autores consiste en convertir el escenario en una ventana abierta a la realidad, a la vida. De ahí que los espectadores asistan a un desfile de personajes que pasean o cruzan por el parque/escenario. La obra adolece de un optimismo ingenuo muy querido por los miembros del grupo, pues la gran mayoría de sus piezas transmiten la alegría de vivir.

Barrio del Este, estrenada el 7 de septiembre de 1946, es la primera comedia en solitario de Alfonso Paso, quien la define como «drama de ambiente en un 
acto». El barrio al que alude el título es el East Side de Nueva York. Como ya sucedió en Un día más, el autor insiste en la misma tesis: el amor y la ilusión vencen a la idea práctica y materialista de la vida.

En esta ocasión, a los ya habituales actores y actrices hay que añadir el nombre de Encarna Paso, sobrina de nuestro autor y cinco años más joven que él: la muchacha tenía solo quince años cuando intervino en esta pieza como hija de un mendigo judío, al que su novio maltrataba.

La obra está marcada por la ingenuidad característica de todas las piezas de Arte Nuevo y, en cierto modo, recuerda las gratas comedias de Charles Chaplin o Frank Capra que, por aquellas fechas, llenaban los cines. El final trae a la memoria Luces de la ciudad, pero sobre todo La quimera del oro en la que el falso mendigo se casa con la auténtica pobre sin saber esta que su novio es realmente rico.

Conocemos de la existencia de un título: La honrada familia Frank; una comedia escrita en colaboración con José Gordón y cuyo estreno estaba anunciado para el día 3 de marzo de 1947 en el teatro María Cristina; sin embargo, nunca llegó a subir a los escenarios.

3 mujeres 3, estrenada en el Instituo Ramiro de Maeztu, el 9 de enero de 1948, es la primera obra humorística de Alfonso Paso, quien la define como «Farsa para hombres en un acto, en prosa y original, aunque con alguna influencia». El propio autor se reservó uno de los protagonistas de comedia, junto a Pilar Peña, Gloria Nieto y $\mathrm{M}^{\mathrm{a}}$. Luisa Romero.

Se trata de una obra misóginamente provocativa que defiende la tesis de que la mujer es un ser poco fiable: únicamente la mujer abnegada y solícita es una buena esposa.

El toque festivo recorre toda la pieza de modo que da pie para la autocrítica. Dice el personaje de El Autor: «Conozco una preciosa definición de baile. Baile, manera inmoral de hacer el idiota. Existe un modo más moral de hacerlo: es tratar de renovar el teatro español» (p. 107).

¿La tesis de la obra? La principal: hacer sonreír al público e, indirectamente, disparar contra la moral burguesa y bienpensante. Entre chanzas y aforismos machistas, el Autor critica la emancipación femenina así como la filosofía existencialista proveniente de Francia.

La última pieza de Alfonso Paso dentro del grupo Arte Nuevo fue Compás de espera, estrenada en el Instituto Cardenal Cisneros el 13 de febrero de 1948 y calificada como drama en un acto. El propio comediógrafo aparece en uno de los personajes principales, acompañado por Amparito Conde, José $\mathrm{M}^{\mathrm{a}}$. Platero y Amparo Romero. En esta ocasión el tema de la obra es el Destino, o su versión cristiana, Dios, que castiga al escéptico y orgulloso, pero premia al creyente. 
La pieza se sitúa en la sala de espera de una estación de tren, que recuerda al parque de Un día más e incluso anticipa obras más ambiciosas como Cuando se espera (1967), de Pedro Laín Entralgo: desfilan por el andén ciertos pasajeros que pretenden representar (y sintetizar) algunos estratos sociales: el matrimonio burgués; la esposa dominante y el marido pusilánime; la joven emancipada; el exitoso hombre de negocios orgulloso por haberse hecho a sí mismo; el donjuán sin escrúpulos; la esposa que anhela nuevas experiencias en las que realizarse plenamente.

La obra, que en su inicio tiene todas las trazas de una pieza realista, toma un giro simbólico con la aparición del personaje Ese Hombre, personificación del Destino, y se convierte en un drama de crimen y venganza.

La labor de Alfonso Paso dentro del grupo no se limitó a la de autor (en solitario o en colaboración), sino que también abarcó otras facetas como la de director o actor. Dirigió El segador, de Azorín, y sus propias obras: 3 mujeres 3 y Compás de espera. Como actor intervino en 3 mujeres 3 y Compás de espera; en El hermano, de Medardo Fraile; en Cargamento de sueños y en Uranio 235, ambas de Alfonso Sastre; y en Tres variaciones sobre una frase de amor, de José María Palacio.

Sobre las dotes de Alfonso Paso como actor, José María de Quinto comentó: «Con todo, debo subrayar las condiciones histriónicas de Alfonso Paso. Y no solo sus espléndidas condiciones histriónicas como actor, sino también como notable director escénico. [...] En todos los casos dio buena prueba de su talento» (en García Lorenzo 1999: 68).

El día 22 de marzo de 1948, en el Instituo Cardenal Cisneros, tuvo lugar la octava y última sesión del grupo de teatro Arte Nuevo. El profesor Mariano de Paco escribía al respecto (1989: 1.078):

Arte Nuevo no consiguió hacer realidad sus propósitos. Las dificultades con las que tropezó lo impidieron porque quizá sus planteamientos no eran en ese momento hacederos. Pero el grupo nos dejó el entusiasmo y la entrega juveniles, ciertas obras estimables que ellos mismos representaron y, lo que es más interesante, el ejemplo de que era posible y necesario trabajar de otro modo en el teatro.

La pasión juvenil por revolucionar el panorama teatral todavía duraría en muchos de los componentes de Arte Nuevo tras su desaparición como grupo. Una vez disuelto, algunos siguieron en el mundo del teatro. José Gordón y José María de Quinto fundaron el grupo teatral La Carátula y José Franco continuó dirigiendo obras en diversas agrupaciones. En lo que respecta a Alfonso Paso y a Alfonso Sastre, ambos ingresaron en el Teatro Universitario de Ensayo 
(TUDE), ligado a la Facultad de Filosofía y Letras de la Universidad de Madrid, bajo la dirección del novelista Jesús Fernández Santos y de Florentino Trapero.

El 29 de marzo de 1949, en la Facultad de Filosofía y Letras de la Universidad Complutense, el TUDE ofreció una sesión teatral semejante a las del extinto Arte Nuevo: tres piezas inéditas en un acto, Mientras cae la lluvia, de Jesús Fernández Santos; Cuando llegue la noche, de Carlos José Costas y Cena para dos, de Alfonso Paso.

Carecemos del texto de la obra de Alfonso Paso, pues nunca fue impreso. Sabemos que se trataba de un drama, de una pieza «seria», según la califica Julio Mathias (1971) y que estuvo protagonizada por Amparito Conde, Ángeles Montenegro, Justo Sanz y el propio Alfonso Paso, que también la dirigió. La obra, según Alfredo Marqueríe (1960: 100), «muestra los extremos a que puede llegar la pasión amorosa desatada de una mujer frente a la indiferencia y al egoísmo masculino». Tras las consultas pertinentes no hemos podido encontrar ninguna crítica sobre la comedia. Tampoco disponemos del expediente de censura.

Tras desavenencias con algunos miembros de los grupos universitarios, Sastre, Paso y varios amigos fundan el grupo teatral La Vaca Flaca que puso en escena tres piezas breves de Tennessee Williams. Su intento de representar La casa de Bernarda Alba chocó con la oposición de los censores. Sin embargo, el grupo teatral La Carátula tuvo mejor suerte y estrenó la obra de Federico García Lorca en el Parque Móvil Ministerial el 20 de marzo de 1950.

Concluida la aventura dentro de los grupos teatrales universitarios, Alfonso Paso comienza a colaborar con Juan Guerrero Zamora en la formación del teatro de cámara El Duende, que funcionó únicamente durante tres sesiones entre marzo y junio de 1950, alquilando el Teatro Gran Vía. El público estuvo formado por abonados que llegaron a alcanzar la cifra de 1.500 personas. José Luis Alonso y el propio Alfonso Paso fueron los directores de escena.

Ligazón, de Valle-Inclán, fue el debut de Alfonso Paso como director en esta compañía: el día 6 de marzo de 1950. La crítica de Alfredo Marqueríe (ABC, 7 de marzo, p. 27) calificó la dirección de Alfonso Paso como «inteligente» y la de José Luis Alonso como «magistral». Unos días después, el 15 de marzo, Manuel Halcón dedicaba la mitad de la tercera del $A B C$ al proyecto de El Duende.

Unos meses más tarde, el 8 de mayo, Alfonso Paso volvía a asumir la dirección teatral, esta vez con la obra El ladrón de niños, de Jules Supervielle. No nos resistimos a reproducir un extenso comentario de Alfonso Paso (Marqueríe, 1960: 21-3), donde el dramaturgo recuerda el ambiente de los ensayos de la obra:

Anales, 29-30 (2018), pp. 231-243 
Como director de escena, aparte de las condiciones precisas, me faltaba la dosis de paciencia franciscana que hay que poseer para tal oficio. Me aburren enormemente los ensayos. A los de mis comedias acudo porque si no, se enfadan conmigo... Cuando se tiene confianza en un montador, bastaría asistir a los cinco o seis últimos ensayos. Escribir una comedia y encima soportarla durante quince o veinte días seguidos me parece una prueba demasiado cruel para cualquier ser humano, aunque éste sea el autor.

[...] Con El ladrón de niños, el implacable juego de las vanidades [...] hizo su aparición y contribuyó a malograr aquella tierna, delicada y notable pieza.

[...] Con un reparto de actores y actrices desunidos en su mayoría, poco podía hacer yo. La obra se estrenó y, contra mis temores, no fue protestada; pero la representación transcurrió en medio de una frialdad absoluta.

La aventura de El Duende terminó cuando la compañía se fusionó con La Carátula para actuar en provincias durante el verano de 1950.

El nombre de Alfonso Paso, unido otra vez al de José Luis Alonso, apareció en un proyecto frustrado: la creación a cargo del Instituto de Humanidades (ligado a los filósofos Julián Marías y José Ortega y Gasset) de un teatro de cámara que iba a llamarse El Espectador. Alfonso Paso y José Luis Alonso serían los directores de escena. El primer montaje previsto, Un hombre como los demás, de Salacrou, nunca llegó a realizarse.

En 1951, nuestro autor obtuvo la licenciatura en Filosofía y Letras, con premio extraordinario en la especialidad de Historia de América. No obstante, la vinculación con el mundo teatral universitario todavía va a durar unos años más.

La comedia en un acto, Yo, Eva, se estrenó el 2 de abril de 1952 en el teatro Infanta Beatriz y se convirtió en primera pieza de una trilogía que, en otro lugar, hemos denominado «trilogía de fantasmas» — completada en años posteriores por las comedias en tres actos: No sé dice adiós, sino hasta luego y 48 horas de felicidad-. Fue puesta en escena por los miembros del TEU de la Escuela Superior de Comercio, donde un jovencísimo Juanjo Menéndez realizaba su primer papel. Ese mismo año, a finales de noviembre, triunfaría con el Dionisio de Tres sombreros de copa, de Miguel Mihura.

El tema de la obra, como el de la trilogía de la que forma parte, es la posibilidad de lo imposible o, si se prefiere, la materialización de los sueños.

La acción tiene lugar en la habitación de un hotel donde Carlos, un comediógrafo, se dispone a pasar solo la Nochebuena. En la radio, que lo acompaña en tan señalada noche, escuchamos que la emisión está dedicada a «los seres que tienen que pasar la Nochebuena solos, a los fracasados, a los desangelados, a los solitarios, a los que arrastran una derrota sentimental». Se remata con el deseo de que Dios traiga compañía a esos seres y (casi parafraseando a Mark 
Twain) se recuerda que donde una mujer y un hombre se encuentran crece siempre un paraíso.

Una mujer con una manzana en la mano entra en la habitación de Carlos por el ventanal de la terraza. Se entabla un gracioso diálogo entre los dos, que supone el núcleo de la comedia. Al final de la obra, Carlos habla con la recepción del hotel y allí le dicen que la mujer que lo acompañó en la Nochebuena no ha existido nunca. Pero mientras Carlos finge estar convencido por estos razonamientos, aprieta entre sus manos la manzana que la mujer le dejó como recuerdo y afirma: «En una Nochebuena hay que esperar, esperar, porque siempre sucede algo maravilloso. En Nochebuena cualquier milagro puede realizarse». El crítico del diario Ya la definió como «una deliciosa comedia breve, llena de gracia ligera y de tierna poesía que se oye con vivo placer».

Y llegamos ya al último contacto de Alfonso Paso con el teatro universitario español. Menos de dos meses después de que el Teatro Popular Universitario, bajo la dirección de Gustavo Pérez Puig, pusiera en escena Escuadra hacia la muerte, de Alfonso Sastre, el mismo grupo escenificó el 6 de mayo de 1953, en el teatro Nacional María Guerrero, Una bomba llamada Abelardo, calificada por su autor como «farsa burlesca, menos fantástica de lo que parece, en dos actos». Algunos de los actores que pusieron sobre las tablas el drama de Sastre también protagonizaron esta comedia de humor ácido y absurdo: Juanjo Menéndez, Agustín González y Fernando Guillén; a los que se unieron Laly Soldevilla, Carmen Lozano, José María de Prada, Margarita Muñiz y Manuel Aleixandre, entre otros, hasta completar un elenco de quince personajes.

Tras su estreno, los críticos pronto la emparentaron con las obras de Tono y Miguel Mihura (Ni pobre ni rico sino todo lo contrario o Tres sombreros de copa). Una bomba llamada Abelardo recrea la disparatada historia de un científico premiado que, en realidad, es un gorila que se escapó cuando era una cría y lo trasladaban a España desde Guinea Ecuatorial. El tal Abelardo ingresará en la universidad y construirá una bomba potentísima que se disputarán soviéticos y norteamericanos. La obra muestra un mundo donde el progreso consiste en destruirnos los unos a los otros: la Guerra Fría y la amenaza nuclear son evidentes bajo las chanzas y las burlas que trufan la comedia.

La obra de Alfonso Paso es también una defensa de la poesía y de la ilusión frente a los convencionalismos sociales, a la seriedad y al belicismo imperante en la época. Para apuntalar el tema principal, Alfonso Paso echa mano de otros temas secundarios: 
a) la crítica a la seriedad personificada sobre todo en los existencialistas franceses y los jóvenes españoles que los imitaban.

MANUEL: No. No transijo con ese tipo. Me cargan los listos, los hombres serios, los feos y tontos que se las dan de profundos. Me carga esa profundidad de moda. Me carga Jean-Paul Sartre, Europa y esta cultura de periódico semiclandestino. Me cargan los mitos, los genios, todos esos tipos que charlan de la guerra sin haber pisado un frente (p. 47).

b) La crítica al belicismo de la época.

LAURENCIA: En lugar de pensar en frivolidades, debías imitarle y hacer algo serio.

MANUEL: Por ejemplo, destruir Europa en treinta y cinco segundos. JONÁS: ¡No hombre, no! Eso al fin y al cabo es la civilización (p. 20).

ABELARDO: Inútil. No entregaré la fórmula de mi nueva arma. Tengo que perfeccionarla. Ahora solo puede matar a dos millones de hombres por explosión. Con trabajo, fe y decisión podrá, Dios mediante, matar a cinco millones de hombres (p. 37).

En la casa de Jonás se prepara el enlace entre Laura y Abelardo. En un ambiente que recuerda a las primeras comedias de Miguel Mihura y a ciertas películas de Frank Capra (Vive como quieras, 1938) y de H. C. Potter (Loquilandia, 1941), Manuel, el hijo de la casa, se opone a las convenciones sociales que representan su madre y su hermana. No entiende cómo todos pueden adorar a Abelardo, un ser horriblemente feo al que la humanidad considera un científico extraordinario.

En medio de un cúmulo de excentricidades —Jonás caza en el salón; agentes norteamericanos y soviéticos se disputan las invenciones de Abelardo-, aparece Laura, una maestra de piano, de la que se enamora locamente Manuel. Ambos representan el lirismo que intenta sobrevivir ante tanta seriedad, hipocresía y belicismo.

En el último acto se descubre que Abelardo no es realmente un ser humano, sino un orangután huido. La crítica contra la ceguera de una sociedad que se mueve por impulsos inconscientes y por modas irreflexivas es evidente en la figura de este personaje que recuerda, en ciertos aspectos, al protagonista de Como mejor están las rubias es con patatas (1947), de Enrique Jardiel Poncela.

Los personajes son meros símbolos: encarnación y personificación de actitudes vitales o de tesis, y actúan como tales. Los ejemplos más evidentes son los representantes norteamericano y soviético, empleados como parodias exageradas de sus respectivos países. Lo cierto es que, al describirlos, Alfonso Paso no se aleja mucho de otros que lo habían hecho antes - Ernst Lubistch 
en Ninotchka (1939)_ o que lo harán más tarde —Billy Wilder en Uno, dos, tres (1961).

Un día antes del estreno, en $A B C$, Alfonso Paso se expresaba de esta guisa en su Autocrítica:

El T.P.U. me ha brindado la ocasión de hacer una farsa burlesca, audaz, combativa [...] Una bomba llamada Abelardo es, ante todo, una obra cómica, al menos yo creo que se halla en la línea del más clásico y limpio humorismo español. Así, puede tener tanto de Quevedo como de Jardiel Poncela. Mi farsa es un modesto alegato contra los mitos que aprisionan Europa en la hora actual, contra toda esa serie de términos oscuros, de filosofías difíciles y de calderilla pagada a peso de oro. Es, pues, una obra con «tesis». Pese a esto, a mí me divierte mucho y espero que a ustedes les divierta también.

Alfonso Paso está convencido de que a través de la seriedad y de la trascendencia no se conseguirá cambiar la sociedad, todo lo más conseguir una úlcera. La crítica a la posición existencialista —que ya habíamos hallado en obras anteriores- supone, en cierta medida, un ataque a la ideología teatral de algunos de sus amigos como Alfonso Sastre o José María de Quinto. Para Alfonso Paso la «solución francesa» no es válida, pues acrecienta el desasosiego del ser humano y se regodea en las miserias.

Futuras creaciones de Miguel Mihura, como Mi adorado Juan, o del propio Alfonso Paso, en El canto de la cigarra, acentúan dicha actitud antisocial, de desprecio por un mundo materialista donde se desdeña y se rechaza el lirismo.

En líneas generales las críticas fueron amables — porque Una bomba llamada Abelardo es una obra amable - y alabaron la originalidad del planteamiento y, en cierto modo, la valentía de la tesis propuesta. Todos, de un modo u otro, recordaron las intenciones de Arte Nuevo y también el estreno de Tres sombreros de copa, un año antes y por la misma compañía teatral.

La consulta del expediente de censura arroja curiosos datos sobre la opinión de los censores. En primer lugar, la obra se presentó a la censura el día 3 de mayo de 1953, es decir, únicamente tres días antes del estreno. La solicitud fue firmada por Jesús López Varela-Álvarez de Castro, Jefe Nacional del Sindicato Español Universitario (SEU). Era práctica habitual presentar los originales con tan poco tiempo antes del estreno, de ese modo se pretendía influir en la opinión del censor al mostrarle que una prohicibión supondría un contratiempo enorme tanto artística como económicamente. Esta medida de presión no siempre surgía el efecto deseado, pues algunos censores mostraban su mala fe y tardaban a la hora de dictaminar, de modo que la obra debía aplazar su estreno. En el caso que nos ocupa, la medida dio resultado y el censor se apremió. Adolfo Carril —que así firmó el lector de la obra- la autorizó el día 4 de mayo si se suprimía una página y algunas oraciones sueltas que se habían 
marcado con tinta roja sobre el original mecanografiado, como estas líneas del Acto II que dice el personaje de Laura: «Me quisieron casar con un señor que tenía blancos los pelos del pecho. [...] Manolo tiene los pelos del pecho negros». El censor puso tres condicionantes a la autorización:

a) La obra deberá representarse en sesión única y nocturna.

b) Solamente tendrán acceso personas mayores de edad pertenecientes al TEU o aquellas a las que se proporcionen localidades.

c) La acción habrá de situarse en el extranjero. En consecuencia, todos los nombres españoles o cualquier referencia a lugares de acción enclavados en territorio nacional habrán de modificarse.

Advertimos también que en el original figuraba una cita de Aristófanes que en la edición publicada se suprimió: «La burla es también un modo de vencer el mal. Y aquel que desprecia la burla, desprecia, en cierto modo, la virtud».

El censor, que desconocía la máxima que hace alusión a la capacidad destructora del humor, cerró su informe, y con ello cerramos también esta intervención, con el siguiente comentario bastante ingenuo: «Su carácter extremadamente burlesco le resta peligrosidad a todos los órdenes».

\section{Bibliografía citada}

Cantos Casenave, Marieta y Romero Ferrer, Alberto (eds.) (2003), La comedia española: entre el realismo, la provocación y las nuevas formas (1950-2000), Universidad de Cádiz y Fundación Pedro Muñoz Seca.

Fraile, Medardo, (ed.) (1994³), Teatro español en un acto (1940-1952), Madrid, Cátedra.

García LoRenzo, Luciano (1975), El teatro español hoy, Barcelona, Planeta.

García Lorenzo, Luciano (ed.) (1999), Aproximación al Teatro Español Universitario, Madrid, C.S.I.C.

García Ruiz, Víctor y ToRres Nebrera, Gregorio (eds.) (2003), Historia y antología del teatro español de posguerra (1940-1975). Vol. II: 1945-1950, Madrid, Fundamentos.

- (2006), Historia y antología del teatro español de posguerra (1940-1975). Vol. III: 1950-1955, Madrid, Fundamentos.

García TemPlado, José (1980), Literatura de la postguerra: El teatro, Madrid, Cincel.

Gordón, José (ed.) (1949), Teatro de Vanguardia. 15 obras de Arte Nuevo, Madrid, Perman.

- (1965), Teatro experimental español (Antología e historia), Madrid, Escelicer.

GUERRERO ZAMORA, Juan (1961-1967), Historia del teatro contemporáneo, IV volúmenes (I: 1961; II: 1961; III: 1962; IV: 1967), Barcelona, Juan Flors-Editor. 
Huerta CAlvo, Javier (ed.) (2003), Historia del teatro español. II. Del siglo XVIII a la época actual, Madrid, Gredos.

Marqueríe, Alfredo (1960), Alfonso Paso y su teatro, Madrid, Escelicer.

MATHIAS, Julio (1971), Alfonso Paso, Madrid, EPESA.

PACO, Mariano de (1989), «El grupo “Arte Nuevo" y el teatro español de postguerra», en Estudios Románicos, número 5, Murcia, Universidad de Murcia, pp. $1.065-1.078$.

- (2003), «El primer teatro de Alfonso Paso», en Cantos Casenave, Marieta y ROMERO FERrER, Alberto (2003), pp. 171-185.

PASO, Alfonso (1953), Una bomba llamada Abelardo. Madrid, Escelicer.

PAYÁ Beltrán, José (2015), Alfonso Paso y el teatro español durante el franquismo, Tesis doctoral, Repositorio de la Universidad de Alicante (RUA). http://hdl. handle.net/10045/52050

Ruiz RAmón, Francisco (1971), Historia del teatro español. Tomo 2. Siglo XX, Madrid, Alianza.

SAnZ VillanueVA, Santos $\left(1991^{4}\right)$, Historia de la literatura española 6/2. Literatura actual, Barcelona, Ariel. 